\title{
Monatshefte für Praktische Dermatologie: Dermatologische Wochenschrift - Dermatologische Monatsschrift - Zeitschrift für Dermatologie - Aktuelle Dermatologie 1882-2004
}

H. Meffert

\section{Zusammenfassung}

Das erste Exemplar der Monatshefte für Praktische Dermatologie erschien im März 1882. Die Zeitschrift war als Erste ausdrücklich der Weiterbildung verpflichtet. Das bedeutete einen wichtigen Schritt in der Herausbildung und Festigung des eigenständigen Fachgebiets Haut- und Geschlechtskrankheiten. Die ersten Herausgeber waren $\mathrm{H}$. von Hebra (Wien), O. Lassar (Berlin) und P. G. Unna (Hamburg). Neben den vorbildlich knappen und präzisen Weiterbildungsartikeln dürften den heutigen Leser insbesondere Erstbeschreibungen von Krankheitsbildern und erstaunlich modern anmutende Beobachtungen an gesunder und kranker Haut interessieren.

Seit Januar 1912 erschien die Zeitschrift unter dem neuen Namen Dermatologische Wochenschrift. Trotz wechselvoller Zeiten und Bezeichnungen gelang es den Herausgebern immer wieder, die Zeitschrift als einen Ort der postgradualen Weiterbildung und der wissenschaftlichen Diskussion zu erhalten.

Im Jahre 1969 wurde die Zeitschrift zum Offiziellen Organ der Gesellschaft für Dermatologie der Deutschen Demokratischen Republik ernannt und in Dermatologische Monatsschrift umbenannt. Nach der Wiedervereinigung Deutschlands wechselte der Name nochmals. Seit 1994 hieß sie Zeitschrift für Dermatologie. 1999 ging die Zeitschrift in Aktuelle Dermatologie auf.

\section{Abstract}

The first issue of Monatshefte für Praktische Dermatologie appeared in March 1882. From the beginning on the journal was dedicated to practical dermatology. Its foundation was an important step in the process of defining and strengthening dermatology as a medical speciality. The founding editors were $\mathrm{H}$. von Hebra (Vienna), O. Lassar (Berlin) and P. G. Unna (Hamburg). Besides the predominating educational papers many first descriptions of skin diseases and nowadays more recently looking observations on healthy and diseased skin had been published. Since January 1912 the journal appeared weekly under the new name Dermatologische Wochenschrift. In spite of changing times and names the publishers again and again succeeded in preserving the journal as a place of postgradual education and scientific exchange. In 1969 the journal was named Dermatologische Monatsschrift and became the official organ of Dermatologische Gesellschaft der Deutschen Demokratischen Republik. After German reunification the name was changed again in 1994, now into Zeitschrift für Dermatologie. In 1999 it merged with Aktuelle Dermatologie.

\section{Einleitung}

Im März 1882 erschien das erste Exemplar der Monatshefte für Praktische Dermatologie, der ersten ausdrücklich auf die hautärztliche Praxis ausgerichtete Zeitschrift. Redigiert wurde diese Zeitschrift für Dermatologie und Syphilislehre von H. von Hebra (Wien), O. Lassar (Berlin) und P. G. Unna (Hamburg). Verlegt wurde sie von Leopold Voss, Hamburg und Leipzig. Der Druck erfolgte bei Metzger \& Wittig in Leipzig.

Das älteste wissenschaftliche Archiv unseres Fachgebiets war das 1866 gegründete Giornale italiano delle malattie veneree e della pelle. Ihm folgte im Jahre 1869 das von H. Auspitz (Wien) und F. 

fiip.

\section{Wraktilase Aermutolonie}

redigiert von

\author{
Dr. H. v. Hebra \\ Wien.
}

Dr. 0. Lassar

Berlin.

Dr. P. G. Unna Hambury.

Band I.

No. 1 .

März 1882.

DERMATOLOGISCHE WOCHENSCHRIFT

FRÜHER: MONATSHEFTE FÜR PRAKTISCHE DERMATOLOGIE



20. Januar 1912

Nr. 3

Band 54

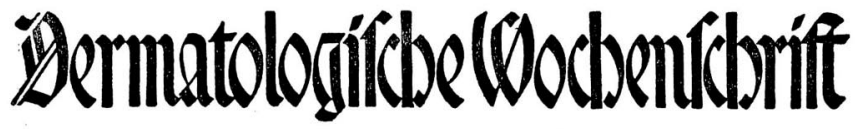

Bd. 100, Nr. 1

5. Januar 1935

DERMATOLOGISCHE

WOCHENSCHRIFT

\section{Dermatologische Monatsschriit}

\section{Zeitschrift für

 und deren Grenzgebiete}

Abb. 1 Titelzeilen.

J. Pick (Prag) herausgegebene deutschsprachige Archiv für Dermatologie und Syphilis (Abb. 1, 2).

Monatshefte für Praktische Dermatologie Konzept und Meriten

Die Gründung der Monatshefte war ein wichtiger Schritt bei der Herausbildung und Festigung des eigenständigen Fachgebiets Haut- und Geschlechtskrankheiten. Das nur als Torso gedruckte Lehrbuch der Hautkrankheiten von E. W. F. von Baerensprung (1859) und mehr noch das von F. von Hebra (1860) hatten wesentlich zur Konsolidierung beigetragen. Die Monatshefte für

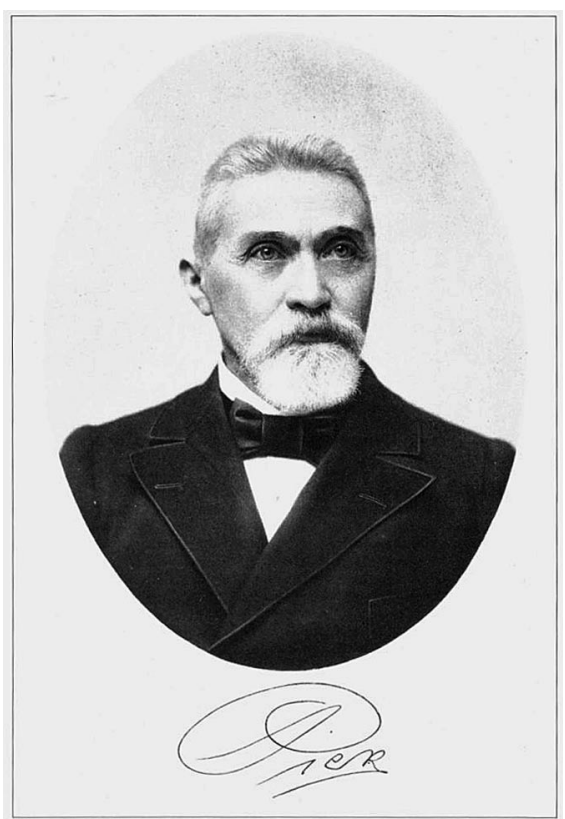

Abb. 2 Filipp Josef Pick (1834-1910).

Bild: Stüttgen G.

(Hrsg). Standort und Ausblick der deutschsprachigen Dermatologie. Zum 100-jährigen Bestehen der Deutschen Dermatologischen Gesellschaft. Berlin: G. Grosse, 1989.

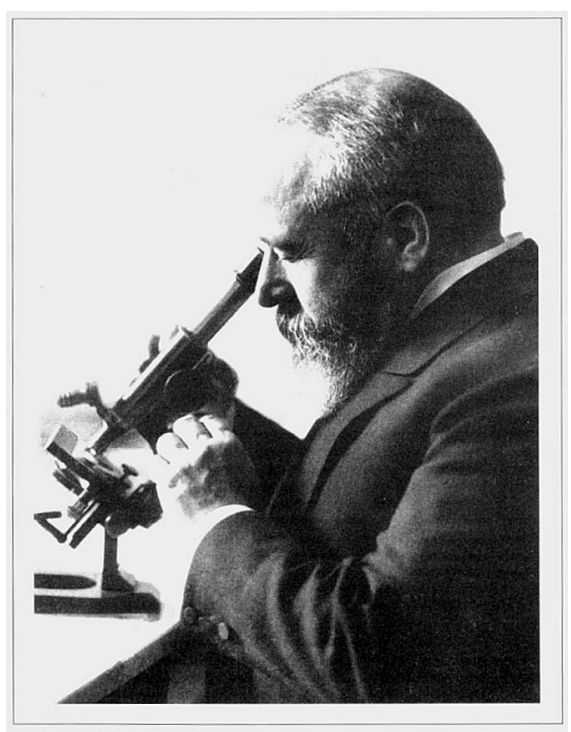

Abb. 3 Paul Gerson Unna (1850 - 1929) beim Mikroskopieren. Bild: s. Abb. 2.

Praktische Dermatologie sollten den praktizierenden Dermatovenerologen rasche und qualifizierte Weiterbildung ermöglichen. Den Herausgebern stand auch damals ein umfangreiches und internationales Gremium ständiger Mitarbeiter zur Seite, dem viele Doktoren, einige Privatdozenten und auffällig wenige Professoren angehörten.

Zwei einleitende Sätze des ersten Heftes seien in vollem Wortlaut zitiert: „Die vorliegenden Monatshefte wollen den mit Dermatologie und Syphilis beschäftigten Ärzten Gelegenheit bieten zur Veröffentlichung kurz gehaltener Mitteilungen, deren Schwerpunkt weniger in Vorführung einer weitschichtigen Literatur und der Entwicklung theoretischer Anschauungen, als vornehmlich in der Berücksichtigung praktisch verwertbarer Erfahrungen und Gesichtspunkte liegen soll... In Rücksicht auf die für umfassende Arbeiten bestimmte Vierteljahresschrift für Dermatologie und Syphilis müssen wir jedoch bitten, den uns zugedachten Mitteilungen einen $1 / 4$ Bogen übersteigenden Umfang nicht 
geben zu wollen“ [1]. Auch wurde tunlichste Gedrungenheit der Darstellung angemahnt. In wieweit diese Bemerkungen auch als Seitenhieb auf das Archiv für Dermatologie und Syphilis zu verstehen waren sei dahingestellt. Außer Originalmitteilungen wurden in den Monatsheften vor allem Referate und Übersichten „über die wertvolleren Veröffentlichungen aller Sprachen“ angekündigt. Dieses Feld war großzügig definiert und umschloss auch Referate über aus heutiger Sicht eher unterhaltsame, venerologisch bis kulturpolitisch und philologisch ausgerichtete Artikel, beispielsweise den von Henri Fournier über die Lügen der Reklame und den Diebstahl in den Pissoirs im Heft 9-10 des Journal des Maladies cutanées et syphilitiques von 1909. Darin wurde beklagt, dass einige Kollegen auf Anschlägen in Pissoirs behauptet hatten, Tripper und Syphilis innerhalb weniger Tage heilen zu können.

Neben den vorbildlich knappen und präzisen Weiterbildungsartikeln dürften den heutigen Leser insbesondere Erstbeschreibungen und scharfsinnige Exkurse wie die des im Gründungsjahr erst einunddreißigjährigen P. G. Unna interessieren. „Die Salizylsäure ist u.a. ein sicheres Mittel, um die normale oder pathologisch verdickte Hornschicht in beliebiger Ausdehnung als eine fest zusammenhängende, nicht abblätternde, weißlich verfärbte Membran schmerzlos abzuheben“" [2]. Die Abhebung findet in der Hornschicht in um so größerer Tiefe statt je stärker die Konzentration des salizylsäurehaltigen Mittels und je dicker die Hornschicht ist.

Auf den folgenden zwei Seiten ist die Erstbeschreibung des Quincke-Ödems zu lesen. Einer exakten Beschreibung der Symptomatik folgt ein offenbar zeitloser Hinweis. „Für die Behandlung... gewährte in einigen Fällen Regulierung der Lebensweise, namentlich der Verdauung prophylaktischen Nutzen“ [3].

Im gleichen Band berichtete A. Goldschneider vom 22-jährigen Musketier K., dessen Füße nach jedem Marsch mit Blasen übersät waren, die sich in der Stachelschicht gebildet hatten, sich nachfolgend exsudativ veränderten, ansonsten auch durch längeres Reiben erzeugt werden konnten und auch bei mehreren Blutsverwandten beobachtet worden waren [4]. Nur eines der vielen Glanzlichter ist die Abgrenzung der Dermatitis herpetiformis durch L. A. Duhring [5].

Leider kann hier aus Platzgründen (sic!) von vielen weiteren, kurz und knackig präsentierten Manifestationen dermatologischen Forschergeistes nicht berichtet werden. Von Anfang an bestand aber auch eine Tendenz zu umfangreicheren Artikeln, wie sie dann in Dermatologische Wochenschrift immer häufiger erschienen.

\section{Dermatologische Wochenschrift}

Ab Januar 1912 wurde die Zeitschrift nach einem gewandelten Konzept gestaltet. Das Fach Dermatologie hatte sich etabliert, was einen sichtbaren Ausdruck in der Errichtung erster Ordinariate, für A. Neisser in Breslau (1907) und E. Lesser in Berlin (1911), gefunden hatte [6]. In der prosperierenden Zeit vor dem Ersten Weltkrieg schien wöchentliches Erscheinen angemessen. Als Herausgeber fungierten neben Altmeister P. G. Unna (Hamburg) die deutlich jüngeren J. H. Rille (Leipzig) und E. Delbanco

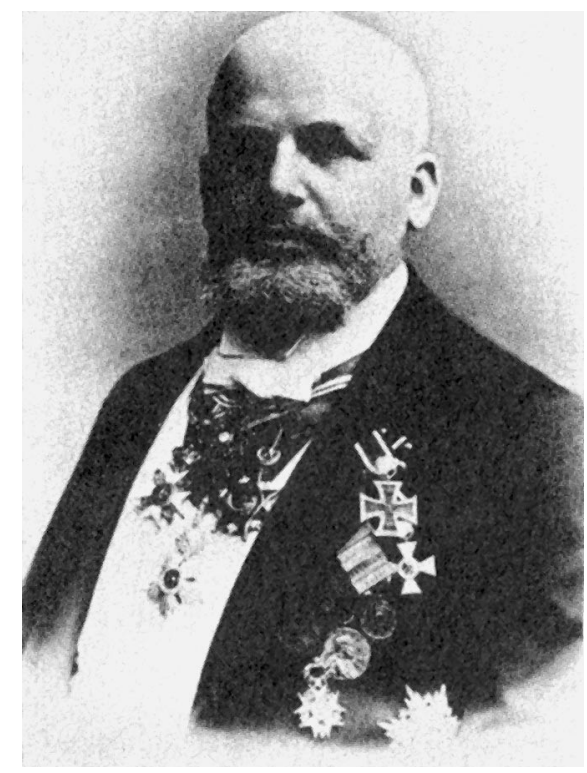

Abb. 4 Oscar Lassar (1849-1907). Bild aus: Klaschka NE (Hrsg). Hundert Jahre Dermatologie in Berlin. Grosse Verlag Berlin, 1984.

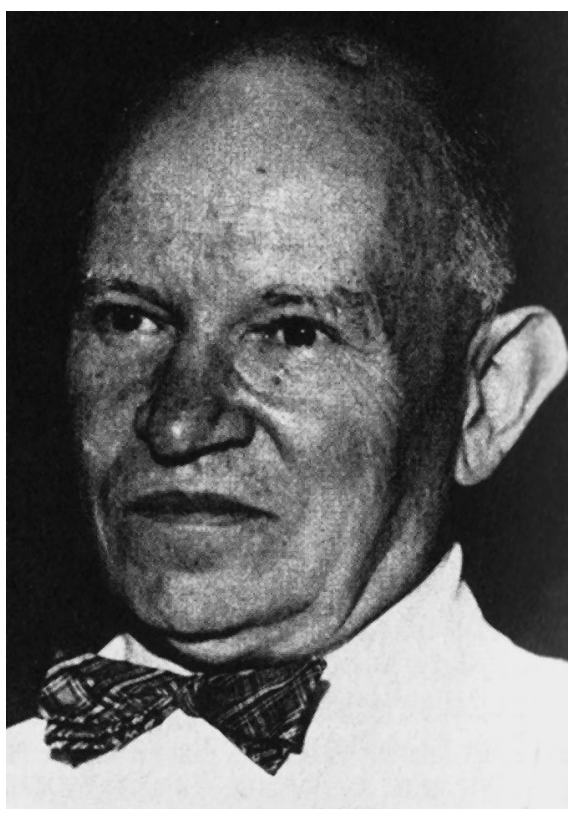

Abb. 5 Egon Keining (1892-1971) Bild: s. Abb. 4

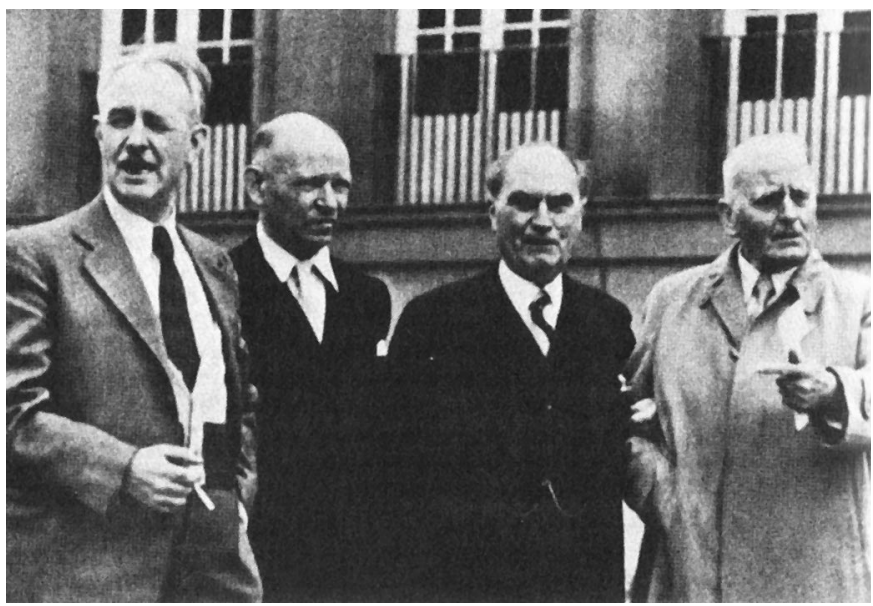

Abb. 6 Heinrich A. Gottron (1890-1974), Egon Keining (18921971), Erich Hoffmann (1868 - 1959) und Heinrich Löhe (1877-1961) um 1950 in Berlin (von links nach rechts). Bild: s. Abb. 4. 
(Hamburg). „[Es] ... verlangt heute der schnelle Fortschritt der Wissenschaft ein allwöchentliches Erscheinen ... Wir rechnen aber auch ganz besonders auf die Mitarbeit der Vertreter der vielen anderen Disziplinen (wie der Chemie, Physik, Bakteriologie usw.), deren Unterstützung die moderne Dermatologie nicht entbehren kann ...“ [7].

Diesen einleitenden Worten an die verehrte Leserschaft folgte Kapitel IX der Serie „Zur Chemie der Haut“ [8]. Hier wurden Vorgänge in der Haut beschrieben, die heute durch Schlagworte wie angeregter Sauerstoff, freie Radikale und Lipidperoxidation allgemein bekannt geworden sind. Die Serie „Zur Chemie der Haut“ ist Geburts- und Sternstunde der Histochemie der Haut. Ihre Lektüre sei dermatologischen Jungforschern als unerlässlich ans Herz gelegt.

Während die Zeitschrift den Ersten Weltkrieg weitgehend glimpflich überstanden hatte, fügten Nationalsozialismus und Zweiter Weltkrieg auch ihr schweren Schaden zu [9]. Rassistische Artikel finden sich selten: „Die bei den ungarländischen $\mathrm{Zi}$ geunern durchgeführten Untersuchungen stellten fest, dass zwischen den nichtzigeunerischen Einwohnern eine Volksgruppe eingekeilt lebt, die nicht nur ein Schmarotzer des Wirtsvolkes ist, sondern diese durch ihre hochgradige syphilitische Durchseuchung ständig bedroht" [10]. 1944 stellte die Zeitschrift mit Band 118 ihr Erscheinen ein. Erst 1948 erschien sie wieder im Leipziger Verlag Johann Ambrosius Barth. Herausgeber waren $\mathrm{H}$. Löhe (Berlin), C. Moncorps (Münster i. W.), J. Hämel (Jena), J. H. Rille (Innsbruck), H. A. Gottron (Tübingen) und W. Schönfeld (Heidelberg). Trotz Kalten Krieges und divergierender Ideologien gelang es den Herausgebern und seit 1961 dem alleinverantwortlichen Redakteur E. Keining (Mainz) erstaunlicherweise, die Vereinnahmung durch die Herrschenden 20 Jahre lang zu verhindern. In der durch einen schwierigen wirtschaftlichen Wiederaufbau und politische Drangsalierung gekennzeichneten Nachkriegszeit war die Dermatologische Wochenschrift auch ein Ort allseitiger, mit der Dermatologie verbundener Bildung: „Heuer im Goethejahr geziemt es sich wohl daran zu erinnern, dass unser erhabenster Genius Goethe vor 163 Jahren am 11. oder 12. September 1786 bei seiner Durchfahrt durch Tirol an der deutsch-italienischen Sprachgrenze eine neue Krankheit entdeckt hat, die nicht zum wenigsten auch dem dermatologischen Bereich angehörige Pellagra und vor allem ihre Ursache" [11] (Abb. 5, 6).

\section{Dermatologische Monatsschrift}

Politischer Druck war es, der 1964 zur Gründung einer Sektion Dermatologie der Deutschen Gesellschaft für klinische Medizin in der DDR geführt hatte, aus welcher bald danach die Gesellschaft für Dermatologie der DDR hervorgegangen war. Zum Jahresbeginn 1969 schieden alle bisherigen Herausgeber der Dermatologischen Wochenschrift unfreiwillig aus. Die Zeitschrift wurde ab Band 155 (1969) als Dermatologische Monatsschrift mit dem Zusatz „Organ der Dermatologischen Gesellschaft der Deutschen Demokratischen Republik“ weitergeführt. Herausgeber waren W. Gertler (Berlin), H. E. Kleine-Natrop (Dresden) und N. Sönnichsen (Jena). Formal änderte sich sonst wenig. „Die Zeitschriftenreferate laufen aus, da diese in der Regel so spät eingehen, dass sie bei Erscheinen keinen aktuellen Wert mehr besitzen“ [12]. Hinter dieser nicht unvernünftig klingenden Ankündigung verbarg sich der im Medienzeitalter untaugliche Versuch, das unkontrollierte Eindringen von Informationen in den eigenen Herrschaftsbereich behindern zu wollen. Dieses Vorhaben scheiterte schon an den vielen Kongressankündigungen und -berichten, die oft durch H. E. Kleine-Natrop (Dresden) inauguriert waren. Zeitweilig wurde nach konspirativer Absprache zwischen $\mathrm{O}$. Braun-Falco (München) und N. Sönnichsen (Berlin) der Kongresskalender des Hautarzt nur wenig verfremdet abgedruckt. Der seit dem 1. 1. 1976 allein verantwortliche Chefredakteur N. Sönnichsen (Berlin) wurde wegen der Ankündigung westdeutscher Veranstaltungen mehrfach ermahnt. An der Publikationspraxis änderte sich nichts.

Im Rahmen des Möglichen entwickelte sich die Dermatologische Monatsschrift anders als von der Politik vorgesehen. Eine rege wissenschaftliche Publikationstätigkeit, Übersichten, originäre Kolumnen wie „Dermatologische Arbeitsempfehlungen“ oder „Randbemerkungen zum Dermatologischen Zeitgeschehen“, die aus heutiger Sicht oft geradezu verklärten Reise- und Kongreßberichte aus der großen weiten Welt, die nach wie vor abgedruckten Tagungsberichte einiger - im politischen Sinne - westdeutscher Fachgesellschaften und die kontinuierliche Pflege deutschsprachiger Artikel aus Ländern wie Ungarn, Polen, Tschechoslowakei, Bulgarien, Rumänien oder Georgien stärkten das Fachgebiet und wirkten der angestrebten Isolierung entgegen.

\section{Zeitschrift fürr Dermatologie - Aktuelle Dermatologie}

Die deutsche Wiedervereinigung und die in ihrem Gefolge aufkommenden Turbulenzen des - im politischen Sinne - ostdeutschen wissenschaftlichen Publikationswesens gingen auch an dieser Zeitschrift nicht spurlos vorbei. Als Herausgeber wurden im Band 179 (1993) N. Sönnichsen (Berlin), C. Orfanos (Berlin), W. Sterry (Ulm) und H. H. Wolff (Lübeck) genannt. Noch im gleichen Jahr wurde der Verlag Johann Ambrosius Barth und mit ihm

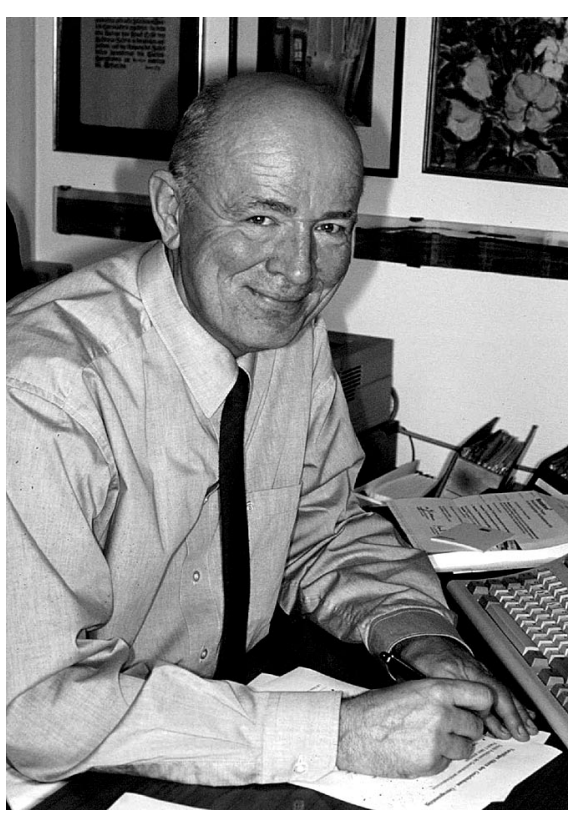

Abb. 7 Hans Meffert (geb. 1938). 
die Dermatologische Monatsschrift von der Verlagsgruppe Hüthig übernommen. Diese vermittelte die Zeitschrift an den Verleger Jürgen Hartmann (Klebheim, Oberfranken). Die Schriftleitung oblag von Band 180 (1994) bis Band 184 (1998) zunächst H. Meffert (Berlin, Abb. 7), W. Sterry (Ulm), M. Wengenroth (Wiesbaden) und H. H. Wolff (Lübeck), dann H. Meffert (Berlin) und M. Wengenroth (Wiesbaden). Die Namensänderung in Zeitschrift für Dermatologie und deren Grenzgebiete sollte veränderte Erscheinungsweise und thematische Erweiterung signalisieren [13]. Die Neuorientierung der Zeitschriftenlandschaft im wiedervereinigten Deutschland führte dazu, dass die Zeitschrift für Dermatologie im Jahre 1999 in der jetzt von E. G. Jung (Mannheim) herausgegebenen Aktuelle Dermatologie aufging.

\section{Literatur}

1 von Hebra H, Lassar O, Unna PG. An die Leser! Monatshefte Prakt Dermatol 1882; $1: 1-2$

${ }^{2}$ Unna PG. Eine besondere Eigenschaft der Salicylsäure. Monatshefte Prakt Dermatol 1882; 1: 128 -

${ }^{3}$ Quincke H. Über akutes umschriebenes Hautödem. Monatshefte Prakt Dermatol 1882; 1: 129-13

${ }^{4}$ Goldschneider A. Hereditäre Neigung zur Blasenbildung. Monatshefte Prakt Dermatol 1882; 1: $163-164$

${ }^{5}$ Duhring LA. Über die Abgrenzung der Dermatitis herpetiformis. Monatshefte Prakt Dermatol 1888; 7: 158-173

${ }^{6}$ Harnack K. Die Hautklinik an der Charité und die Dermatologie in Berlin (1710 - 1999) Berlin: Berliner Medizinische Verlagsanstalt GmbH, 2000

7 Unna PG. An unsere Leser. Dermatol Wochenschr 1912; 54: 1 - 2

${ }^{8}$ Unna PG, Golodetz L. Zur Chemie der Haut. IX. Die Verteilung des Sauerstoffs und der Sauerstoffermente in der Haut. Dermatol Wochenschr 1912; 54: $2-9$

${ }^{9}$ Scholz A. Geschichte der Dermatologie in Deutschland Berlin, Heidelberg, New York: Springer-Verlag, 1999

${ }^{10}$ von Szentkirályi S. Beiträge zur Frage der Infektion der Zigeuner mit Syphilis. Dermatol Wochenschr 1942; 116: 591 -600

${ }^{11}$ Rille JH. Gedenktage der Dermatologie. Dermatol Wochenschr 1949; 120: $559-560$

${ }^{12}$ Herausgeber und Verlag. Zum Geleit. Dermatol Monatsschr 1969; 155: $1-2$

${ }^{13}$ Hartmann J. Editorial. Z Dermatol 1994; 180: 3 